\title{
On Extremal Behavior of Gaussian Chaos
}

\author{
D. A. Korshunov ${ }^{a}$, V. I. Piterbarg ${ }^{b}$, and E. Hashorvac \\ Presented by Academician A.N. Shiryaev March 6, 2013
}

Received March 22, 2013

DOI: $10.1134 / \mathrm{S} 1064562413050220$

Let $\xi=\left(\xi_{1}, \xi_{2}, \ldots, \xi_{d}\right)$ be a normally distributed random vector in $\mathbb{R}^{d}$ with zero mean and covariance matrix $B, B_{i j}:=\mathbb{E} \xi_{i} \xi_{j}$. A problem of great interest is to analyze the asymptotic behavior of the distribution tail of the product $\prod_{i=1}^{d} \xi_{i}$. This problem arises in various domains, for example in stochastic geometry, random difference equations, and risk theory.

Consider a more general case of functions of the vector $\xi$, namely, the so-called Gaussian chaos $h(\xi)$, where $h: \mathbb{R}^{d} \rightarrow \mathbb{R}$ is a continuous homogeneous function of order $\alpha>0$; i.e., $h(x \mathbf{t})=x^{\alpha} h(\mathbf{t})$ for all $x>0$ and $\mathbf{t} \in \mathbb{R}^{d}$. Traditionally, in the literature, the term Gaussian chaos of order $\alpha \in \mathbb{N}$ is referred to the case where $g$ is a homogeneous polynomial of degree $\alpha$. This concept goes back to Wiener [14], who was the first to consider processes of polynomial chaos. We follow a broader treatment of the concept of Gaussian chaos.

The distribution of $\xi$ is equal to the distribution of $\sqrt{B} \boldsymbol{\eta}$ if the vector $\boldsymbol{\eta}=\left(\eta_{1}, \eta_{2}, \ldots, \eta_{d}\right)$ has independent coordinates with a standard normal distribution. Then

$$
\begin{gathered}
\mathbb{P}\{h(\xi)>x\}=\mathbb{P}\{h(\sqrt{B} \boldsymbol{\eta})>x\} \\
=\mathbb{P}\{g(\boldsymbol{\eta})>x\},
\end{gathered}
$$

where $g(\mathbf{u})=h(\sqrt{B} \mathbf{u})$. The continuous function $g$ : $\mathbb{R}^{d} \rightarrow \mathbb{R}$ is also homogeneous of order $\alpha$ like $h$. Thus, the problem is reduced to the case of a unit covariance matrix. For this reason, in what follows, we study $g(\boldsymbol{\eta})$. By virtue of homogeneity,

\footnotetext{
a Sobolev Institute of Mathematics, Siberian Branch, Russian Academy of Sciences, pr. Akademika Koptyuga 4, Novosibirsk, 630090 Russia

${ }^{b}$ Faculty of Mechanics and Mathematics, Moscow State University, Moscow, 119992 Russia

${ }^{c}$ UNIL-Dorigny, 1015 Lausanne, Switzerland e-mail:korshunov@math.nsc.ru,piter@mech.math.msu.su, enkelejd.hashorva@unil.ch
}

$$
\begin{gathered}
\mathbb{P}\{g(\boldsymbol{\eta})>x\}=\mathbb{P}\left\{\left(g\left(x^{-1 / \alpha} \boldsymbol{\eta}\right)>1\right\}\right. \\
=\frac{x^{d / \alpha}}{(2 \pi)^{d / 2}} \int_{\{\mathbf{v}: g(\mathbf{v})>1\}} e^{-x^{2 / \alpha}|\mathbf{v}|^{2} / 2} d \mathbf{v} .
\end{gathered}
$$

Therefore, the asymptotic behavior of probability (1) can be determined using a version of the Laplace asymptotic method (see, for example, [3]). Define

$$
\begin{gathered}
c^{2}:=\min \left\{|\mathbf{u}|^{2}: g(\mathbf{u}) \geq 1\right\} \\
\quad=\min \left\{|\mathbf{u}|^{2}: g(\mathbf{u})=1\right\},
\end{gathered}
$$

where the last equality follows from the homogeneity of $g$. Since $g$ is continuous, we have $c^{2}>0$. To apply the Laplace method, we consider the set

$$
\begin{aligned}
& \mathscr{C}:=\arg \min \{|\mathbf{u}|: g(\mathbf{u})=1\} \\
= & \{\mathbf{u}:|\mathbf{u}|=c \text { and } g(\mathbf{u})=1\},
\end{aligned}
$$

which lies on a sphere of radius $c$. Assume that this set is a smooth finitely connected manifold of dimension $r$ and the structure of the function $g$ near this manifold is typical of the Laplace method. Define $g(\varphi):=$ $g(\mathbf{u} /|\mathbf{u}|)$, where $\boldsymbol{\varphi}=\left(\varphi_{1}, \varphi_{2}, \ldots, \varphi_{d-1}\right) \in \Pi:=[0, \pi)^{d-2} \times$ $[0,2 \pi)$ are the spherical coordinates of the vector $\mathbf{u} /|\mathbf{u}|$ on the unit sphere $S_{d-1}$. The manifold on the parallelepiped $\Pi$ that corresponds to $\mathscr{C}$ is denoted by $\mathscr{C}_{\varphi}$. The Jacobian of the transition to spherical coordinates in $\mathbb{R}^{d}$ is designated as $J(r, \varphi)$. Let $g^{\prime \prime}(\varphi)$ denote the Hessian of a function $g(\varphi)$, and let $\lambda(A)$ stand for the smallest (in absolute value) nonzero eigenvalue of a symmetric matrix $A$.

Theorem 1. Let $g: \mathbb{R}^{d} \rightarrow \mathbb{R}$ be a continuous homogeneous function of order $\alpha>0$, and let $\operatorname{dim} \mathscr{C}_{\varphi}=r \in$ $[0, d-1]$. If the corresponding function $g(\varphi): \Pi \rightarrow \mathbb{R}$ is three times differentiable and

$$
\operatorname{rank} g^{\prime \prime}(\varphi) \equiv d-1-r, \quad \inf _{\varphi \in \mathscr{C}_{\varphi}} \lambda\left(g^{\prime \prime}(\varphi)\right)>0
$$

(the Hessian is uniformly nonsingular on $\mathscr{C}_{\varphi}$ ), then

$$
\begin{gathered}
\mathbb{P}\{g(\boldsymbol{\eta})>x\}=\mathscr{H} x^{(r-1) / \alpha} e^{-c^{2} x^{2 / \alpha} / 2}\left(1+O\left(x^{-2 / \alpha}\right)\right) \\
\text { as } x \rightarrow \infty,
\end{gathered}
$$




$$
\begin{aligned}
\mathscr{H} & :=\frac{1}{(2 \pi)^{(r+1) / 2}} \frac{\alpha^{(d-1-r) / 2}}{c^{1-r+\alpha(d-1-r) / 2}} \\
& \times \int_{\mathscr{C}_{\varphi}} \frac{J(1, \boldsymbol{\varphi})}{\sqrt{\left|\operatorname{det}_{d-1-r}^{\prime \prime}(\varphi)\right|}} d V_{\varphi},
\end{aligned}
$$

where $d V_{\varphi}$ is the volume element of the manifold $\mathscr{C}_{\varphi} \subset \Pi$ and $\operatorname{det} g_{d-1-r}^{\prime \prime}(\varphi)$ is any nonzero minor of the Hessian $g^{\prime \prime}(\varphi)$ of order $d-1-r$. Relation (2) can be differentiated, which gives asymptotics of the distribution density of the Gaussian chaos $g(\boldsymbol{\eta})$.

Note that, as in the classical case of the Laplace method [3], assuming that $g$ has higher smoothness, we can obtain asymptotic expansions of the considered probability and density in powers of $x$. In the case $r=0$, i.e., when $\mathscr{C}=\left\{\mathbf{t}_{1}, \mathbf{t}_{2}, \ldots, \mathbf{t}_{k}\right\}$, where $\mathbf{t}_{i}$ are isolated absolute minimizers of $g$ in the integration domain, the theorem is proved by directly applying Theorem 4.2 from [3]. The integral in the expression for $\mathscr{H}$ becomes a sum over the points $\varphi_{i} \in \Pi$ corresponding to the points $\mathbf{t}_{i}$. In the general case, we apply a version of the Laplace method for parameter-dependent functions, which are used to prove the possibility of integration. On each map of an atlas with sufficiently small maps on the manifold $\mathscr{C}_{\varphi}$, we construct a coordinate system with the first $r$ coordinates being parameters. When they are fixed, the minimum of the amplitude (of the argument of the exponential) is reached at a unique point of a neighborhood of the map. Next, the standard Laplace method is applied and the maps of the atlas are integrated with respect to these parameters on all neighborhoods in $\Pi$.

By Theorem 1, the Gaussian chaos is a subexponential random variable if $\alpha>2$. The subexponentiality of random variables is an important concept in various applications (see, for example, [4]). The Gaussian chaos is subexponential under rather weak constraints on the function $h$. For example, let $h$ be nonnegative. The $d$-dimensional centered Gaussian vector $\boldsymbol{\eta}$ with a unit covariance matrix can be represented as the product $\boldsymbol{\eta} \stackrel{d}{=} \chi \boldsymbol{\mu}$ of independent values $\chi$ and $\boldsymbol{\mu}$, where $\chi^{2}=\sum_{i=1}^{d} \eta_{i}^{2}$ has a chi-square distribution $\chi^{2}$ with $d$ degrees of freedom, while $\boldsymbol{\mu}$ has a uniform distribution on the unit sphere $S_{d-1} \subset \mathbb{R}^{d}$. The Gaussian random vector $\xi=\sqrt{B} \boldsymbol{\eta}=\chi \sqrt{B} \boldsymbol{\mu}$ has the covariance matrix $B$. Therefore, since $h$ is homogeneous for any $x>0$, we have

$$
\mathbb{P}\{h(\xi)>x\}=\mathbb{P}\left\{\chi^{\alpha} h(\sqrt{B} \boldsymbol{\mu})>x\right\} .
$$

If $h(\sqrt{B} \boldsymbol{\mu})$ is a positive bounded random variable, then, according to [2, Corollary 2.5$]$, the random vari- able $h(\xi)$ is subexponential for $\alpha>2$, because the distribution $\chi^{\alpha}$ then has a Weibull type density

$$
\frac{1}{\alpha \cdot 2^{d / 2-1} \Gamma(d / 2)} x^{d / \alpha-1} e^{-x^{2 / \alpha} / 2}
$$

with $2 / \alpha<1$, which means subexponentiality.

It follows from (3) that, if $h$ is bounded on the unit sphere $S_{d-1}$, i.e., $h^{*}:=\max \{h(\mathbf{u}):|\mathbf{u}|=1\}<\infty$, then estimates

$$
\begin{gathered}
\mathbb{P}\{h(\xi)>x\} \leq \mathbb{P}\left\{\chi^{\alpha}>x / h^{*}\right\} \\
\leq \frac{1}{\alpha \cdot 2^{d / 2-1} \Gamma(d / 2)} \int_{x / h^{*}}^{\infty} y^{d / \alpha-1} e^{-y^{2 / \alpha} / 2} d y .
\end{gathered}
$$

This explicit upper bound improves the one obtained in [10, Corollary 1]. In our conditions, it is better than the bound that can be derived from [1, Theorem 4.3].

Theorem 1 underlies a unified approach to different problems. Below are some examples.

Example 1. (Product of independent $N(0,1)$ random variables) Let $\boldsymbol{\eta}=\left(\eta_{1}, \eta_{2}, \ldots, \eta_{d}\right)$ be a standard Gaussian vector and $g(\mathbf{u})=u_{1} u_{2} \ldots u_{d}$. We have $\alpha=d$, $c^{2}=d$, and $\mathscr{C}=\{( \pm 1, \ldots, \pm 1)$ with an even number of negative coordinates $\}$ consists of $2^{d-1}$ points. Applying Theorem 1 yields the asymptotics

$$
\begin{gathered}
p_{\eta_{1} \ldots \eta_{d}}(x)=\frac{2^{(d-1) / 2}}{\sqrt{2 \pi d}} x^{1 / d-1} e^{-d x^{2 / d} / 2}\left(1+O\left(x^{-2 / d}\right)\right) \\
\text { as } x \rightarrow \infty .
\end{gathered}
$$

This asymptotic relation can be intuitively interpreted as follows (see, e.g., [13]): the product takes the most probable large value when all the multipliers are roughly identical; therefore, $p_{\eta_{1} \ldots \eta_{d}}(x)$ asymptotically resembles the product of $d$ densities at the same point $x^{1 / d}$.

For the product of the coordinates of an arbitrary Gaussian vector $\xi$ with a covariance matrix $B$, we have a similar formula based on the representation $\xi=$ $\sqrt{B} \boldsymbol{\eta}$, but the computation of the constants encounters certain difficulties.

Example 2. (Quadratic forms of independent $N(0,1)$ random variables.) Let $g(\boldsymbol{\eta})=\sum_{i=1}^{d} a_{i} \eta_{i}^{2}$, where the constants $a_{i} \in \mathbb{R}$ are such that $a_{1} \leq a_{2} \leq \ldots \leq a_{d-r}<$ $a_{d-r+1}=\ldots=a_{d}=a, a>0$.

Since

$$
g(\mathbf{u})=\sum_{i=1}^{d-r} a_{i} u_{i}^{2}+a \sum_{i=d-r+1}^{d} u_{i}^{2}
$$

and $a_{i}<a$ for $i \leq d-r$, the minimum of $|\mathbf{u}|^{2}$ on the set $g(\mathbf{u})=1$ is reached at points $\mathbf{u}$ satisfying $u_{d-r+1}^{2}+\ldots+$ $u_{d}^{2}=\frac{1}{a}$ and $u_{1}=u_{2}=\ldots=u_{d-r}=0$, so that $c^{2}=\frac{1}{a}$. If 
$r=1$, the set $\mathscr{C}_{\varphi}$ consists of two points $\left(\frac{\pi}{2}, \ldots, \frac{\pi}{2}, \frac{\pi}{2}\right)$

and $\left(\frac{\pi}{2}, \ldots, \frac{\pi}{2}, \frac{3 \pi}{2}\right)$. By using Theorem 1 , we can find that

$$
\begin{gathered}
\mathbb{P}\left\{\sum_{i=1}^{d} a_{i} \eta_{i}^{2}>x\right\} \\
=\frac{1}{2^{r / 2-1} \Gamma(r / 2)} \prod_{i=1}^{d-r} \frac{1}{\sqrt{1-a_{i} / a}}(x / a)^{r / 2-1} e^{-x / 2 a}(1+O(1 / x))
\end{gathered}
$$

as $x \rightarrow \infty$, which agrees (up to the first-order asymptotics) with the results of [6] (see also [11, 12] or [7, Theorem 1]). This also supplements the upper bounds obtained in [5, 9].

Example 3. (Scalar product) The quadratic forms in Example 2 are closely related to $g\left(\boldsymbol{\eta}, \boldsymbol{\eta}^{*}\right)=$ $\sum_{i=1}^{d} a_{i} \eta_{i} \eta_{i}^{*}$, where $\eta_{i}$ and $\eta_{i}^{*}, i \leq d$, are independent $N(0,1)$ random variables and $a_{i} \in \mathbb{R}^{+}$. Indeed, since $\eta_{i} \eta_{i}^{*}$ coincides in distribution with

$$
\frac{\eta_{i}+\eta_{i}^{*}}{\sqrt{2}} \frac{\eta_{i}-\eta_{i}^{*}}{\sqrt{2}}=\frac{\eta_{i}^{2}-\eta_{i}^{*^{2}}}{2}
$$

we have the distribution equality

$$
g\left(\boldsymbol{\eta}, \boldsymbol{\eta}^{*}\right) \stackrel{d}{=} \frac{1}{2}\left(\sum_{i=1}^{d} a_{i} \eta_{i}^{2}-\sum_{i=1}^{d} a_{i} \eta_{i}^{*^{2}}\right),
$$

and, to the quadratic form on the right, we can apply the result of Example 2, with the dimension replaced by $2 d$ and with the parameter $r$ replaced by the number of maximal $a_{i}$. Some results for scalar products can be found in [8].

\section{REFERENCES}

1. M. Arcones and E. Giné, J. Theor. Probab. 6, 101-122 (1993).

2. D. B. H. Cline and G. Samorodnitsky, Stoch. Process. Appl. 49, 75-98 (1994).

3. M. V. Fedoryuk, Asymptotics: Integrals and Series (Nauka, Moscow, 1987) [in Russian].

4. S. Foss, D. Korshunov, and S. Zachary, An Introduction to Heavy-Tailed and Subexponential Distributions (Springer, New York, 2011).

5. D. L. Hanson and F. T. Wright, Ann. Math. Stat. 42, 1079-1083 (1971).

6. W. Hoeffding, Theory Probab. Appl. 9 (1), 89-91 (1964).

7. J. Hüsler, R. Liu, and K. Singh, J. Multiv. Anal. 82, 422-430 (2002).

8. B. G. Ivanoff and N. C. Weber, Bull. Austral. Math. Soc. 58, 239-244 (1998).

9. R. Latała, Stud. Math. 135, 39-53 (1999).

10. R. Latała, Ann. Probab. 34, 2315-2331 (2006).

11. M. A. Lifshits, Lectures on Gaussian Processes (Springer, Heidelberg, 2012).

12. V. I. Piterbarg, Stoch. Process. Appl. 53, 307-337 (1994).

13. D. Sornette, Phys. Rev. E 57, 4811-4813 (1998).

14. N. Wiener, Am. J. Math. 60, 897-936 (1938).

Translated by I. Ruzanova 